Supporting Information to manuscript :

\title{
Real Time Evaluation of the Composition and Structure of Concanavalin A Adsorbed on Polystyrene Surface
}

Jerzy A. Mielczarski*, Jie Dong and Ela Mielczarski

\section{EXPERIMENTAL SECTION}

\section{Materials}

Polystyrene (PS) with average molecular weight 250,000 g/mol was purchased from ACROS. Concanavalin A (ConA) was purchased from Sigma-Aldrich (C2010, carbohydrate and salt are essentially free) and used directly without further purification. Bovine serum albumin (BSA) (fraction V, lyophilized, purity 95\%) was supplied by Odczynniki Chemiczne, Poland and used as a blocking agent. Phosphate buffered saline (PBS) buffer solution was prepared from $\mathrm{KH}_{2} \mathrm{PO}_{4}(1.47 \mathrm{mM}), \mathrm{Na}_{2} \mathrm{HPO}_{4}(6.46 \mathrm{mM}), \mathrm{KCl}(2.68 \mathrm{mM})$ and $\mathrm{NaCl}(0.137 \mathrm{M}) . \mathrm{NaOH}$ and $\mathrm{HCl}$ were used to adjust the $\mathrm{pH}$. All solutions were freshly prepared before adsorption experiments. ConA concentration was determined by UV/Vis spectroscopy before and after filtration using 0.2 filter. No agglomeration of ConA in solution was detected. All reagents used were of analytical grade. Ultrapure water from the Millipore (Milli-Q system) was used throughout the experiments.

\section{Infrared (FTIR) Spectroscopy}

FTIR spectrometer (Bruker IFS55) equipped with an MCT detector and an attenuated total reflection (ATR) attachment was used to record the ATR spectra. A wire-grid polarizer was placed before the sample and p- or s-polarized light was provided. These accessories were from Harrick Scientific Co. The reflection spectra of the adsorption layers were obtained by the use of polarized light at incidence angle of $45^{\circ}$. The spectrometer was purged with dry air 
(Balston filter) to minimize the disturbance of water vapor and carbon dioxide to the recorded spectra. The unit of intensity was defined as $-\log \left(\frac{R}{R_{0}}\right)$, where $R$ and $R_{0}$ are the reflectivities of the system with and without the investigated adsorption layer, respectively. 200 scans were collected for samples and references spectra. The resolution was $4 \mathrm{~cm}^{-1}$ (zero-filled =2) with Blackman-Harris, three-term apodization.

\section{X-ray Photoelectron Spectroscopy (XPS)}

AXIS NOVA photoelectron spectrometer (Kratos Analytical, Manchester, UK) equipped with monochromatic AlKa ( $h v=1486.6 \mathrm{eV})$ anode was used in the studies. The kinetic energy of the photoelectrons was determined with the hemispheric analyser set to the pass energy of 160 $\mathrm{eV}$ for wide scan spectra and $20 \mathrm{eV}$ for high resolution spectra. Electrostatic charge effect of sample was overcompensated by means of the low-energy electron source working in combination with magnetic immersion lens. The carbon C1s line with position at $284.6 \mathrm{eV}$ was used as a reference to correct the charging effect. Quantitative elemental compositions were determined from peak areas using experimentally determined sensitivity factors and spectrometer transmission function. Spectrum background was subtracted according to Shirley. The high resolution spectra were analyzed by means of spectra deconvolution software (Vision 2, Kratos Analytical, UK).

In order to study the molecular structure of the top surface layers, i.e. a film of a few nanometers thickness, angle resolved XPS was applied and spectra were recorded for the three take-off angles of $\theta=0^{\circ}, 60^{\circ}$, and $75^{\circ}$. The take-off angle is defined as the angle between the normal to the surface of the sample and the electron optical axis of the spectrometer. The effective information depth, varies according to $d=d_{0} \cos \theta$, where $d_{o}$ is the maximum information depth $\left(d_{o} \sim 10 \mathrm{~nm}\right.$ for the $\mathrm{C} 1 \mathrm{~s}$ line by employing an $\mathrm{AlK} \alpha$ source). 
This technique helps to determine structure (molecular distribution) of the adsorbed ConA layer.

\section{Scanning Electron Microscope (SEM) Analysis}

SEM studies were carried out by the use of a HITACHI S-2500 SEM equipped with a spectrometer EDS super Quantum Delta and with a detector of retro-diffused electrons. Scanning electron micrographs were obtained within a large interval of focal amplification.

\section{Preparation of Thin film of Polystyrene for ConA Adsorption}

The measurements of ConA adsorption were done by attenuated total reflection (ATR) infrared method, therefore, the thin film of PS was deposited on germanium ATR reflection element with dimension of $50 \times 20 \times 3 \mathrm{~mm}^{3}$ (16 reflections) and an angle of reflection of $45^{\circ}$. The assembled liquid cell with reflection element is illustrated schematically in Fig. S1.A.

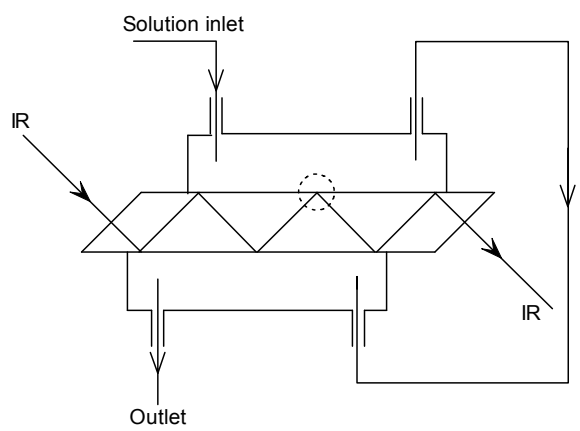

A

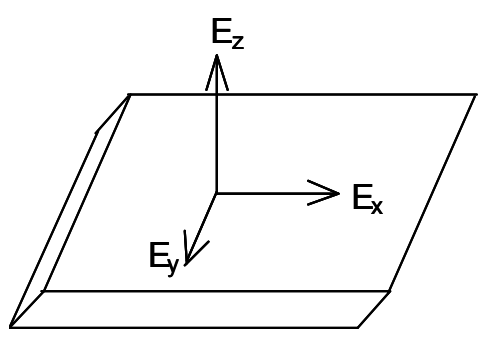

B

Fig. S1. A. Schematic illustration of the assembled Teflon liquid cell with Ge reflection element for in situ adsorption studies by ATR-IR. B. Directions of electric field components at $\mathrm{Ge}$ interface

The reflection crystal was polished with alumina powder $(<0.05 \mu \mathrm{m})$ washed with water and finally with toluene. PS was deposited on both sides of germanium crystal by dip coating from PS toluene solution with concentration of $10 \mathrm{mg} / \mathrm{ml}$ solution. The retracing speed of the 
coating device was equivalent to $18 \mathrm{~mm} / \mathrm{min}$. The produced PS film was characterized by XPS, SEM and infrared spectroscopy. The film was pure PS (with only trace amount of oxygen), the layer was continuous, the surface of PS was smooth (SEM results, not shown) and the thickness of the film, determined by infrared technique combined with spectral simulation (for details see Optical Consideration section), was around $16 \mathrm{~nm}$. Reproducibility of the prepared PS films was $16 \pm 1 \mathrm{~nm}$.

\section{Determination of Number of the Active Internal Reflections}

Number of the active internal reflections of in situ monitoring of protein adsorption could be estimated from geometrical dimensions of liquid cell and reflection element (Fig. S1). However more accurate result can be obtained from the recorded experimental reflection spectra of water in liquid cell.

Two experimental spectra recorded for s- and p-polarizations with germanium reflection element held in liquid cell with and without water allow measurement of water absorbance according to the following expression:

$$
A b s=-\log \left(\frac{R\left(\mathrm{Ge} / \mathrm{H}_{2} \mathrm{O}\right)}{R_{o}(\mathrm{Ge} / \text { air })}\right)
$$

The water absorbance for one reflection can be simulated based on optical constants of reflection element and water for two polarizations at $45^{\circ}$. By simple fitting of the experimental spectra of water in liquid cell with simulated spectra for two polarizations the number of active reflections of in situ measurement was calculated. The number obtained was equal to 12.2 reflections.

\section{Determination of Thickness and Structure of the Deposited PS Layer}

Polystyrene is an excellent substrate used in the study of protein adsorption because of its smooth surface, negligible solubility in water and invariable surface characteristics ${ }^{1,2}$. A lot of 
work has been done concerning the orientation of PS itself by sum frequency generation $(\mathrm{SFG})^{2,3}$, angle-resolved ultraviolet photoelectron spectroscopy (ARUPS) ${ }^{4}$ or near-edge X-ray absorption fine structure (NEXAFS) $)^{5,6}$, small-angle neutron scattering (SANS) ${ }^{7}$. The above mentioned experimental studies reported that the phenyl groups on the PS surface are orientated closely to the surface normal with a narrow angle distribution. Most recently, by molecular dynamics simulation, it was found ${ }^{8}$ that the phenyl rings point outwards at the PS/air or vacuum interface, but rings in the bulk show no preferred orientation. In contrast with the phenyl rings, the bisectors for the methylene groups show little tendency for orientation, even when the methylene groups are close to the surface. In the present work at first approximation it was assumed that PS layer is isotropic.

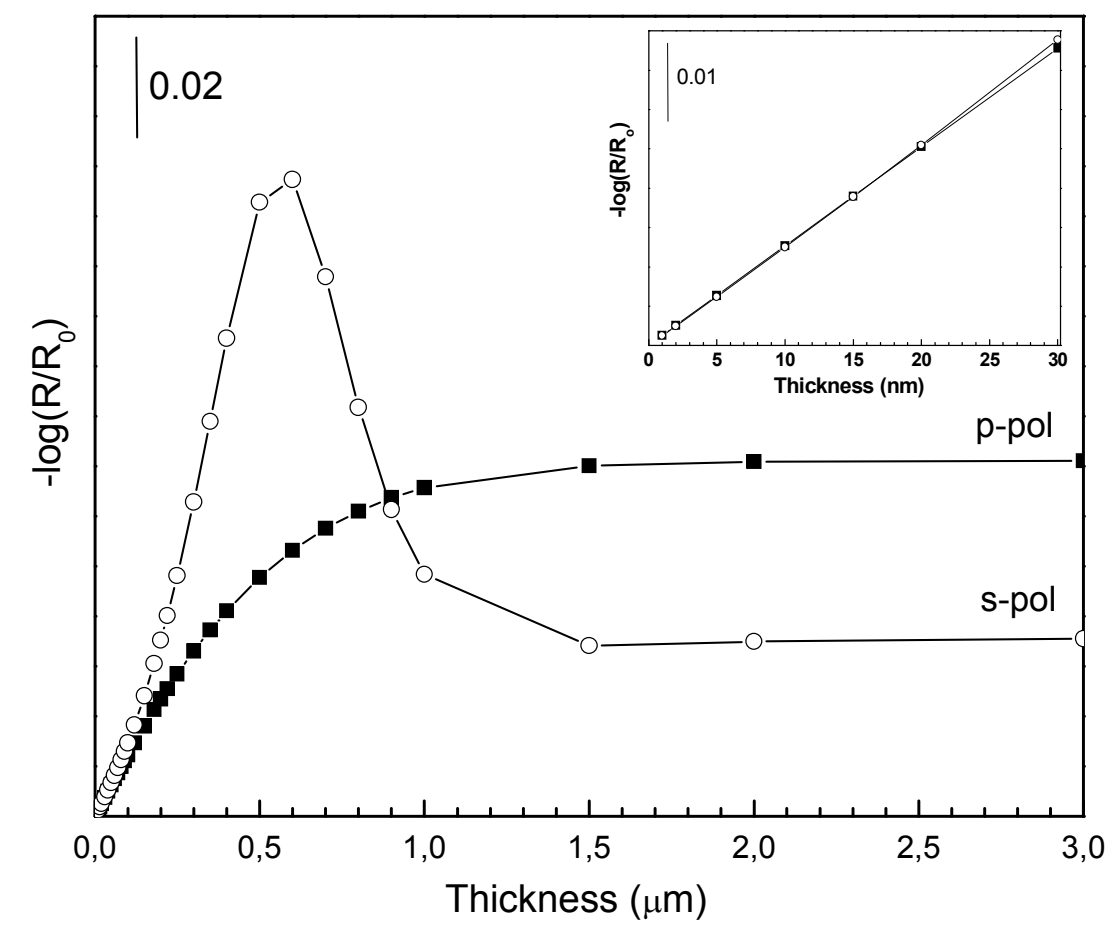

Fig. S2. Simulated absorbance intensity of characteristic PS absorbance band at $1494 \mathrm{~cm}^{-1}$ versus thickness of hypothetical PS isotropic layer for p- (filled square line) and s- (open circle line) polarizations. Simulation was performed for system of $\mathrm{R}(\mathrm{Ge} / \mathrm{PS} / \mathrm{air})$ and $\mathrm{R}_{\mathrm{o}}(\mathrm{Ge} / \mathrm{air})$ 
Results of the intensity simulation of the characteristic PS absorbance band at $1494 \mathrm{~cm}^{-1}$ versus thickness of the deposited layer (Fig. S2) clearly indicate that for isotropic PS layer with thickness up to $20 \mathrm{~nm}$ the reflection spectra recorded for $\mathrm{p}$ - and s-polarizations should be identical. Above this thickness other optical effects take place and the intensity of p-polarized and s-polarized spectra vary significantly with thickness up to $1.5 \mu \mathrm{m}$ (Fig. S2). It should be noted that at thickness above of $1.5 \mu \mathrm{m}$ constant values are observed for both polarizations with absorbance ratio equal to 2:1, which is characteristic for ATR spectrum of massive sample.

In the case of p-polarization at the Ge/PS interface there are two electric field components (Fig. S1.B): the first one vertical to interface in the $\mathrm{z}$ direction, $\mathrm{E}_{\mathrm{Z}}$, and the second one parallel to interface in the $\mathrm{x}$ direction, $\mathrm{E}_{\mathrm{X}}$. For s-polarization there is only electric field component parallel to interface in the $y$ direction, $E_{Y}$. Hence if the PS layer is anisotropic the recorded spectra for both polarizations should be different. Since the ATR experimental spectra of the PS layers recorded in air and in water

$$
A b s=-\log \frac{R(G e / P S / \text { air })}{R_{o}(\mathrm{Ge} / \text { air })} \quad A b s=-\log \frac{R\left(G e / P S / \mathrm{H}_{2} \mathrm{O}\right)}{R_{o}\left(\mathrm{Ge} / \mathrm{H}_{2} \mathrm{O}\right)}
$$

are almost identical for both polarizations (Fig. S3) it implies that PS layer could be considered as an isotropic phase, which simplifies the carried out optical considerations. 


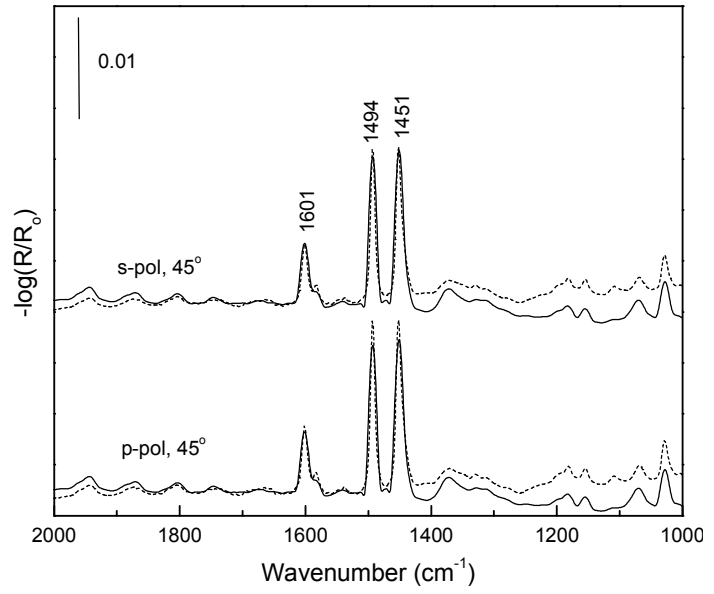

Figure S3. Experimental spectra (dashed lines) of deposited PS layer on Ge reflection element compared with simulated spectra (solid lines) of $16 \mathrm{~nm}$ hypothetical isotropic PS layer for both polarizations.

The best fitting of the experimental spectra recorded in air is presented in Fig. S3, which was achieved with assumption of $16 \mathrm{~nm}$ thick PS layer. It is possible to obtain the PS layers on Ge crystal by dip coating with precision $16 \pm 1 \mathrm{~nm}$.

\section{REFERENCES}

\footnotetext{
${ }^{1}$ Edward D. P. "Handbook of Optical Constants of Solids”, Naval Research Laboratory, Washington, D. C., Academic Press Inc. 1985.

${ }^{2}$ Chen, C.; Wang, J.; Woodcock, S. E.; Chen, Z. Langmuir 2002, 18, 1302-1309.

${ }^{3}$ Gautam, K. S.; Schwab, A. D.; Dhinojwala, A.; Zhang, D.; Dougal, S. M.; Yeganeh, M. S. Phys. Rev. Lett. 2000, 85(18), 3854-3857

${ }^{4}$ Ueno, N.; Azuma, Y.; Tsutsi, M.; Okudaira, K.; Harada, Y. Jpn. J. Appl. Phys. 1998, 37, 4979-4982.

${ }^{5}$ Stöhr, J.; Samat, M. G. J. Elec. Spectroc. Relat. Phenom. 1999, 98-99, 189-207.

${ }^{6}$ Fischer, D. A.; Mitchell, G. E.; Yeh, T. A.; Gland, J. L. Appl. Surf.Sci. 1998, 133, 58-64.

${ }^{7}$ Jones, R. L.; Kumar, S. K.; Ho, D. L.; Briber, R. M.; Russell, T. Nature 1999, 400, 146-149.

${ }^{8}$ Clancy, T.; Jang, J.;H.; Dhinojwala, A. and Mattice, W. J. Phys. Chem. B 2001, 105, 11483 11497.
} 